\title{
Manejo perioperatorio de la medicación crónica no relacionada con la cirugía
}

\author{
R. JUVANY ROIG, G. MERCADAL ORFILA, R. JÓDAR MASANÉS \\ Servicio de Farmacia. Hospital Universitari de Bellvitge. L'Hospitalet de Llobregat, \\ Barcelona
}

\section{RESUMEN}

El correcto manejo de la medicación crónica no relacionada con la cirugía adquiere un papel relevante ya que en todo el mundo millones de pacientes se someten año tras año a intervenciones quirúrgicas. El equipo asistencial debe tener presente la importancia de continuar o suprimir determinados fármacos durante el perioperatorio ya que algunos de ellos se consideran un factor de riesgo en el desarrollo de complicaciones. La cuestión fundamental es diferenciar la medicación necesaria de la innecesaria, o bien perjudicial. Éste es un aspecto complejo y todavía poco estudiado lo cual dificulta en algunos casos la toma de decisiones y conlleva a la coexistencia de diversas tendencias de práctica clínica. En este trabajo se revisan los aspectos que condicionan la suspensión o continuidad de la medicación crónica que no está relacionada con la cirugía y se proporcionan recomendaciones prácticas para el manejo de la misma en base a la bibliografía disponible.

PERIOPERATIVE MANAGEMENT OF CHRONIC MEDICATIONS NOT RELATED WITH SURGICAL PROCEDURES

PALABRAS CLAVE: Periodo perioperatorio. Medicación crónica. Paciente quirúrgico. Anestesia.

\begin{abstract}
The correct management of chronic medications not related with surgical procedures in the perioperative period has a relevant place because each year millions of patients around the world undergo surgical procedures. For this reason the assitencial team should be aware of the importance of continuate or discontinuate determinate drugs during perioperative period because some of them are considered an importat risk factor in the development of complications. The key is to diferentiate necessary from innecessary medication. This is a complex aspect, little studied, which dificults clinical decisions and favours the coexistence of several trends of clinical practice. The purpose of this review is to describe the factors that determinate the continuity or suspension of chronic medications which are not related with surgery in the perioperative period and to provide practice recommendations in lights of available publications.
\end{abstract}

KEY WORDS: Perioperative period. Chronic medications. Surgical patients. Anaesthesia.

Juvany Roig R, Mercadal Orfila G, Jódar Masanés R. Manejo perioperatorio de la medicación crónica no relacionada con la cirugía. An Med Interna (Madrid) 2004; 21: 291-300.

\section{INTRODUCCIÓN}

Cada vez es más frecuente que los pacientes sometidos a intervenciones quirúrgicas se hallen en tratamiento crónico con uno o varios medicamentos que no tienen relación con la patología que ocasiona la cirugía (1-4). En un estudio reciente se estima en un $50 \%$ la proporción de pacientes quirúrgicos que se hallan en esta situación (2). Los factores determinantes de la misma son los avances en los procedimientos quirúrgicos y anestésicos, que los hacen disponibles a pacientes con patologías cada vez más complejas y el envejecimiento de la población que favorecen la existencia de pacientes polimedicados crónicos (2,5-7).

A pesar de los avances, las complicaciones en el período perioperatorio son frecuentes, especialmente las cardiovasculares (4,7-9). En este ámbito, los fármacos no relacionados con la cirugía se consideran un factor de riesgo. El único estudio que cuantifica este aspecto es el realizado por Kennedy y col que apunta que dicha medicación incrementa en un $2,7 \%$ el riesgo relativo de desarrollar complicaciones y que la retira-

Trabajo aceptado: 4 de febrero de 2004

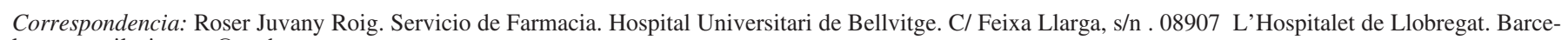
lona.e-mail: rjuvany@csub.scs.es. 
da brusca de algunos fármacos puede incrementar la tasa de complicaciones de forma proporcional al tiempo sin tomar medicación (2).

Es bien conocido que la interrupción brusca de los bloqueantes $\beta$-adrenérgicos puede tener consecuencias equivalentes a las derivadas de un error de medicación, favoreciendo la isquemia miocárdica $(3,4,7,10-12)$. Contrariamente, conviene suprimir fármacos como la aspirina antes de algunas intervenciones quirúrgicas para evitar el riesgo de complicaciones hemorrágicas (13-15). En general, se aconseja suprimir de forma transitoria toda la medicación innecesaria antes de la cirugía por un tiempo entre tres y cinco $t_{1 / 2}$, si es posible (suficiente para eliminar completamente el fármaco y sus metabolitos) (4,13-14). La cuestión clave es diferenciar la medicación necesaria de la innecesaria o bien perjudicial, incluidos los fármacos de venta sin receta, las hierbas medicinales, los suplementos dietéticos y las drogas de abuso $(2,15-$ 23).

El correcto manejo de la medicación crónica no relacionada con la cirugía adquiere un papel relevante ya que en todo el mundo millones de pacientes se someten año tras año a intervenciones quirúrgicas, por lo que es fundamental poder anticiparse a las consecuencias de no suprimir determinados fármacos. Sin embargo, es un aspecto complejo y poco estudiado lo cual dificulta la toma de decisiones. En el caso de intervenciones quirúrgicas programadas es una oportunidad para reexaminar las indicaciones y contraindicaciones del tratamiento y si es necesario cambiarlo; por el contrario, en situaciones de urgencia no hay tiempo para modificar el tratamiento antes de la cirugía $(15,24,25)$. Para algunos fármacos existen recomendaciones de consenso mientras que para otros la información disponible es limitada o controvertida; la mayor parte no procede de ensayos clínicos sino de opiniones de expertos, casos clínicos aislados o consideraciones teóricas extrapoladas a partir de la experiencia obtenida con fármacos similares. Todo ello conlleva la coexistencia de diversas tendencias de práctica clínica $(1,14-16,26-30)$.
En este trabajo se revisan los aspectos que condicionan la suspensión o continuidad de la medicación crónica en el perioperatorio y se proporcionan recomendaciones prácticas para el manejo de la misma en base a la bibliografía disponible con el propósito de proporcionar criterios que permitan unificar la práctica clínica.

ASPECTOS QUE CONDICIONAN LA SUSPENSIÓN O LA CONTINUIDAD DE LA MEDICACIÓN CRÓNICA NO RELACIONADA CON LA CIRUGÍA EN EL PERIOPERATORIO

Los aspectos que condicionan la suspensión o continuidad de la medicación crónica en el perioperatorio son el riesgo de desarrollar complicaciones y los cambios en la función gastrointestinal que pueden imposibilitar la ingesta por vía oral durante varios días $(14,15)$.

\section{FACTORES QUE DETERMINAN EL DESARROLLO DE COMPLICACIONES EN EL PERIOPERATORIO}

\section{Interacción de la medicación crónica no relacionada con la cirugía con los medicamentos administrados durante la inducción y mantenimiento de la anestesia}

Durante este periodo los pacientes se hallan expuestos a nuevas medicaciones durante un tiempo corto pero intenso $(14,31)$. Estos fármacos presentan un elevado potencial de interacciones farmacológicas, especialmente los bloqueantes neuromusculares y algunas de ellas pueden tener consecuencias clínicamente relevantes por lo que es importante anticiparse a las mismas siempre que sea posible (22,26,31-36). En la tabla I se resumen algunos ejemplos de interacciones clínicamente relevantes.

TABLA I

ALGUNOS EJEMPLOS DE INTERACCIONES CLÍNICAMENTE RELEVANTES CON LOS FÁRMACOS ADMINISTRADOS DURANTE LA INDUCCIÓN Y EL MANTENIMIENTO DE LA ANESTESIA

\begin{tabular}{ll}
\hline Interacción & Efecto \\
\hline $\begin{array}{l}\text { Bloqueantes neuromusculares en combinación con } \\
\text { corticoides, inmunosupresores, anti-H2, IBP }\end{array}$ & Antagonizan el bloqueo, disminuyendo la duración del mismo \\
$\begin{array}{l}\text { Bloqueantes neuromusculares en combinación con } \\
\text { aminoglucósidos, polimixinas, lincosaminas, magnesio, } \\
\text { agonistas } \alpha_{2} \text {-adrenérgicos, agonistas } \beta \text {-adrenérgicos, bloqueantes } \\
\text { de los canales del calcio, donepezil }\end{array}$ & Potencian el bloqueo, aumentando la duración del mismo \\
$\begin{array}{l}\text { Dextrometorfano en combinación con antidepresivos IMAO } \\
\text { Enflurano en combinación con antidepresivos tricíclicos }\end{array}$ & Reacciones excitatorias \\
Halotano en combinación con imipramina & Convulsiones \\
Isoflurano o succinilcolina en combinación con anfetaminas & Potencia las arritmias inducidas por adrenalina y pancuronio \\
Petidina en combinación con antidepresivos IMAO & Hipertermia y rabdomiolisis \\
Petidina en combinación con antidepresivos ISRS & Reacciones excitatorias \\
\hline
\end{tabular}

IMAO: Inhibidores de la monoaminooxidasa; anti-H2: bloqueantes de los receptores H2 de la histamina; IBP: inhibidores de la bomba de protones; ISRS: inhibidores selectivos de la recaptación de serotonina. 


\section{Interacción de la medicación crónica con el procedimiento quirúrgico}

Algunos procedimientos quirúrgicos por si solos tienen un elevado riesgo de producir complicaciones hemorrágicas (cirugía mayor, prostatectomia). La aplicación de la anestesia regional (espinal y epidural) puede ocasionar hematomas extradurales, que pueden tener graves consecuencias en pacientes con elevado riesgo de sangrado. En cirugía de retina y neurocirugía las consecuencias del sangrado pueden ser graves aunque este sea mínimo (37).

Algunos anestésicos como halotano, sevoflurano y propofol pueden agravar el riesgo de complicaciones hemorrágicas, ya que inhiben la agregación plaquetar de forma reversible y dosis-dependiente, a las concentraciones utilizadas habitualmente (29).

La cirugía también se asocia con factores que pueden producir fenómenos tromboembólicos como son la inmovilidad y la hipercoagulabilidad sanguínea que acompañan al estrés quirúrgico $(13,26,38,39)$.

La administración crónica de anticoagulantes orales (ej: warfarina y acenocumarol) y fármacos que inhiben la agregación plaquetar (ej: aspirina, antiinflamatorios no esteroideos) puede incrementar el riesgo de sangrado durante la cirugía mientras que la suspensión de los mismos puede incrementar el riesgo de tromboembolismo después de la misma $(13,26,37,38,40,41)$. Igualmente, la administración de anticonceptivos orales combinados (estrógeno más progestágeno) también puede incrementar también el riesgo de tromboembolismo durante el perioperatorio (39).

Para minimizar los riesgos, tanto de sangrado como de tromboembolismo, hay que ajustar el grado de coagulación en función del tipo de cirugía $(13,37,38,40,41)$. En situaciones de emergencia puede ser necesario administrar vitamina $\mathrm{K}$ o hemoderivados (plasma fresco, complejo de protrombina) $(13,38)$.

La pérdida de sangre y fluidos y ocasionalmente el efecto vasoactivo de los anestésicos pueden producir hipotensión. Ésta puede agravarse por los fármacos utilizados en el tratamiento de las enfermedades cardiovasculares (ej: antihipertensivos, antianginosos, antiarritmicos, diuréticos).

El delirio es una complicación frecuente después de la cirugía ortopédica. Los fármacos que se asocian con un mayor riesgo de producirlo son los que presentan efectos anticolinérgicos (amitriptilina, difenhidramina, clorpromazina, doxepina, hidroxicina, imipramina), los narcóticos (meperidina) y los hipnótico-sedantes de larga duración (diazepan). Para prevenir este efecto, los citados fármacos deberían utilizarse a la menor dosis posible, o bien utilizar alternativas más seguras como el haloperidol a bajas dosis o las benzodiazepinas de acción corta (4).

\section{Respuesta fisiológica al estrés producido por la anestesia y la cirugía}

El estrés producido por la anestesia y la cirugía activa el eje hipotalámico-hipofisario-adrenal (EHHA) y el sistema reninaangiotensina, aumenta la secreción de vasopresina, de catecolaminas, de citoquinas y de hormonas como la hormona del crecimiento y la hormona adenocorticotropa (ACTH) que a su vez estimula la secreción de cortisol. Todo ello se traduce en un incremento del catabolismo y en la retención sales y agua para mantener el volumen de fluidos y la homeostasis cardiovascular. La magnitud de los cambios depende del tipo de cirugía, la duración de la misma, el estrés ocasionado y el desarrollo de complicaciones como la sepsis $(23,26,42-46)$. Así, la secreción de cortisol, prácticamente no se modifica después de la cirugía menor mientras que después de cirugía moderada y mayor puede alcanzar niveles de 50-75 mg/día durante 1-2 días y 100-150 mg/día durante 2-3 días, respectivamente (44).

Los cambios metabólicos ocasionados en este período pueden incrementar el riesgo de complicaciones en el paciente en tratamiento crónico con corticoides y en el paciente diabético, por lo que requieren una atención especial $(26,42,44)$.

Los corticoides exógenos pueden suprimir el EHHA inhibiendo la producción endógena de cortisol, de modo que éste puede resultar insuficiente para cubrir los requerimientos del organismo. Los efectos supresores de los corticoides tópicos e inhalados se consideran sin importancia clínica $(4,42-44,47)$. Un déficit de corticoides puede incrementar el riesgo de hipotensión, colapso circulatorio y shock $(13,42)$. A pesar de que existe controversia respecto al tiempo que dura el efecto supresor después de finalizar un tratamiento crónico (de semanas a meses) y que se desconoce la dosis, frecuencia y duración optima del suplemento, se acepta por consenso recomendar la administración de dosis suplementarias de corticoides durante el perioperatorio, para prevenir la potencial y grave complicación derivada de un déficit de los mismos $(4,42-45,47)$. En general, se aconseja individualizar la dosis en función del tipo de cirugía y la dosis habitual preoperatoria $(4,47)$. El objetivo es administrar la dosis mínima eficaz ya que un exceso también puede ocasionar efectos perjudiciales para el organismo como son la hiperglucemia, hipertensión, incremento del metabolismo proteico, retraso en la cicatrización de las heridas, inmunosupresión, estados de psicosis, etc $(4,42-45)$.

En el paciente diabético, la hiperglucemia persistente impide la correcta cicatrización de las heridas, puede aumentar el riesgo de infección local durante el postoperatorio $(40,42)$ y además puede ser la causa de cetosis, acidosis y alteraciones electrolíticas. El manejo perioperatorio de éste paciente requiere garantizar el mantenimiento de la glucemia dentro de los valores de referencia y para ello es necesario optimizar el tratamiento en función de la terapia antidiabética previa, el tipo de diabetes, el tipo de cirugía, la estabilidad de la patología y el grado de afectación orgánica $(40,42,44,48,49)$.

\section{Desestabilización de la patología de base}

La supresión brusca de algunos fármacos puede provocar la reaparición de la patología de base, o bien el síndrome de la retirada. En estos casos, el estrés producido por la cirugía sé superimpone a la patología de base sin su soporte farmacológico habitual por lo que algunos pacientes son más sensibles a dicho efecto. Ello puede dificultar la realización de la cirugía en condiciones óptimas, empeorando el estado del paciente, que queda sometido a más riesgos, posiblemente innecesarios. Algunos ejemplos de fármacos que producen este efecto son 
los bloqueantes $\beta$-adrenérgicos, los agonistas $\alpha$-adrenérgicos, los antidepresivos inhibidores de la monoaminooxidasa (IMAO), los antidepresivos tricíclicos, las benzodiacepinas, los antiepilépticos y los antiparkinsonianos. En general las complicaciones se solventan reintroduciendo la medicación habitual $(2,3,8,10-14,23,26,50)$.

\section{CAMBIOS EN LA FUNCIÓN GASTROINTESTINAL}

La posibilidad de administrar pequeñas cantidades de agua hasta 2 horas antes de la anestesia facilita el mantenimiento de la medicación crónica no relacionada con la cirugía hasta el momento previo a la intervención quirúrgica (26). Después de la cirugía, la vía oral puede quedar temporalmente imposibilitada debido a la aparición de náuseas, vómitos y retraso en el vaciado gástrico, secundarios a la medicación administrada (ej: opioides), debidos al propio procedimiento quirúrgico (ej: cirugía intestinal, de cabeza y cuello), o bien debido a las secuelas del mismo (ej: intubación y/o ventilación mecánica, ileo) (11,13$15,26)$.

En general, los medicamentos que presentan una vida media larga, pueden suprimirse durante varias dosis si es necesario sin provocar efectos indeseables para el paciente (ej: levotiroxina, $\mathrm{t}_{1 / 2}=7$ días; digoxina, $\mathrm{t}_{1 / 2}=36-48$ horas). Hay que tener en cuenta que el $\mathrm{t}_{1 / 2}$ puede estar incrementado en pacientes de edad avanzada ya que su capacidad metabólica y de excreción de fármacos se halla reducida (28). Sin embargo, cuando esta situación se alarga más de 1 ó 2 días se han de utilizar vías de administración alternativas a la vía oral como son la vía intravenosa, la vía rectal, la vía tansdérmica o bien, la sonda nasogástrica. Si el principio activo que se ha de administrar solo se halla disponible por vía oral (ej: antiparkinsonianos), se habrá de sustituir, de forma transitoria, por un fármaco que presente una acción farmacológica similar $(1,28)$. En estas condiciones hay que ajustar las dosis de los nuevos fármacos administrados en función de la respuesta del paciente. Para ello es necesario monitorizar la presión arterial cuando se cambia de antihipertensivo, monitorizar el nivel de glucosa cuando se cambia de antidiabético, o bien monitorizar la concentración sèrica del fármaco si hay que cambiar de antiepileptico, hasta conseguir de nuevo la estabilización del paciente (11).
RECOMENDACIONES PRÁCTICAS PARA EL MANEJO DE LA MEDICACIÓN NO RELACIONADA CON LA CIRUGÍA DURANTE EL PERIOPERATORIO

En el anexo I se resumen las pautas a seguir durante el perioperatorio con algunas de las medicaciones crónicas más habituales, clasificadas por grupo farmacoterapéutico.

\section{CONCLUSIÓN}

La mayor parte de las medicaciones crónicas no relacionadas con la cirugia deben continuarse durante el período perioperatorio sin suponer ningún riesgo adicional para el paciente. No interfieren con los fármacos anestésicos, o la interacción se considera sin importancia clínica, y facilitan la realización de la cirugía en condiciones óptimas como sucede con los medicamentos que actúan sobre el sistema cardiovascular o respiratorio, que contribuyen al mantenimiento de la estabilidad hemodinámica y pulmonar, respectivamente.

Ahora bien, esta práctica no se puede generalizar a todos los fármacos ya que para algunos el beneficio de suspender la medicación supera el riesgo de continuarla como son los anticoagulantes orales, los IMAO o bien, los hipoglucemiantes orales del grupo de las biguanidas en pacientes con insuficiencia renal.

Asimismo, las hierbas medicinales que se han hecho muy populares en los últimos años, deberían suprimirse antes de la cirugía por cuanto sus efectos pueden ser perjudiciales o sencillamente son desconocidos, por lo que es mejor evitarlos.

Finalmente para algunos fármacos, como los IECA, el riesgo continua siendo incierto, lo cual conduce a decisiones individuales, valorando en cada paciente el beneficio-riesgo de continuar o suprimir la medicación. La rapidez con que sé comercialización nuevos fármacos agrava en parte esta situación, porque se desconocen los signos y potenciales secuelas derivadas de la suspensión brusca de los mismos.

Dadas las implicaciones clínicas de esta problemática, es importante que el equipo asistencial tenga presente la importancia de manejar adecuadamente la medicación crónica no relacionada con la cirugia antes de la misma, ya que ello puede contribuir a la reduccción de complicaciones en una fase donde el cuidado del paciente es especialmente crítica, aumentando por consiguiente la calidad del proceso asistencial y disminuyendo su coste económico. 


\section{ANEXO I}

PAUTA A SEGUIR DURANTE EL PERIOPERATORIO CON ALGUNAS DE LAS MEDICACIONES CRÓNICAS MÁS HABITUALES CLASIFICADA POR GRUPO FARMACOTERAPÉUTICO. ¿CONVIENE CONTINUAR LA MEDICACIÓN DURANTE EL PERIOPERATORIO?

Antiinflamatorios no esteroídicos y terapia antirreumática

Inhibidores de la ciclooxigenasa tipo 1

(COX-1): a) de semivida corta (2-5 horas): ibuprofeno, indometacina y b) de semivida larga (12-17 horas):

sulindaco, naproxeno.

Inhibidores de la ciclooxigenasa tipo 2 (COX-2) (rofecoxib, celecoxib).
Metotrexate

\section{Ciclofosfamida}

Azatioprina.
NO. Debido a su efecto inhibidor sobre la agregación plaquetar pueden producir complicaciones hemorrágicas. Pueden alterar la función renal y potenciar la nefrotoxicidad de otros fármacos. Los de semivida corta se suspenden 1 día antes de la cirugía y los de semivida larga 2-3 días antes de la misma $(14,15)$.

Sí, excepto en pacientes con insuficiencia renal (IR) en los que deberían suprimirse 2-3 días antes de la cirugía. Tienen poco o nulo efecto sobre la función plaquetar por lo que el riesgo de hemorragia postquirúrgica está reducido, pero al igual que los inhibidoes de la COX-1 pueden alterar la función renal y potenciar la nefrotoxicidad de otros fármacos. Podrían reducir los requerimientos de opioides y el riesgo de íleo postoperatorio $(14,15,51)$.

Sí, excepto en pacientes con IR en los que debería suspenderse 48 horas antes de la cirugía dado que tiene una excreción renal primaria y genera metabolitos tóxicos que se acumulan rápidamente. Aunque existe controversia en cuanto al incremento del riesgo de infecciones y dificultad de curación de las heridas, se recomienda continuar la medicación $(15,52,53,54,55)$.

NO. Para evitar complicaciones renales o disfunciones de la vejiga tras la intervención quirúrgica, dado que se excreta vía renal (52)

NO. Se ha relacionado con dificultad de cicatrización de las heridas (52).

\section{Terapia cardiovascular}

Antianginosos y antihipertensivos (nitratos, bloqueantes

$\beta$-adrenérgicos, bloqueantes

de los canales del calcio)

\section{Digoxina.}

Antiarrítmicos (amiodarona, quinidina, disopiramida).

Inhibidores del enzima conversora de la angiotensina (captopril) y antagonistas del receptor de angiotensina II (losartan).
Sí. La continuación del tratamiento contribuye al mantenimiento de la estabilidad hemodinámica. En el caso de los pacientes en tratamiento con b-bloqueantes se debe monitorizar el estado de hidratación del paciente, dado que estos fármacos suprimen la respuesta refleja a la hipovolemia $(14,15)$.

sí. Se deben monitorizar los niveles (14).

Sí. Reducen el riesgo de aparición de nuevas arritmias. La amiodarona requiere un control estricto ya que puede producir bradicardia resistente a atropina, toxicidad pulmonar y hepática y vasodilatación intensa $(13,14,38)$. Si el antiarrítmico se utiliza para una alteración menor, como despolarización prematura atrial o ventricular, se recomienda discontinuar la medicación (14).

SEGÚN CRITERIO CLÍNICO. El manejo perioperatorio de estos fárma cos es controvertido. Se ha descrito que pueden mejorar el flujo sanguíneo, el aporte de oxígeno y la función renal en pacientes de riesgo (ej: pacientes con disfunción ventricular izquierda) $(13,38)$. Sin embargo, su continuación se ha asociado con episodios hipotensivos graves de difícil manejo durante la inducción y mantenimiento de la anestesia $(10,56)$.

Sí. Contribuyen al mantenimiento de la estabilidad hemodinámica y minimizan la respuesta al estrés producido por la cirugía. Reducen las dosis de anestésicos y tienen propiedades sedantes, ansiolíticas y analgésicas. Además, su retirada repentina se ha asociado con hipertensión de rebote peligrosa (13-15). 


\section{ANEXO I (CONT.)}

\section{PAUTA A SEGUIR DURANTE EL PERIOPERATORIO CON ALGUNAS DE LAS MEDICACIONES CRÓNICAS MÁS HABITUALES} CLASIFICADA POR GRUPO FARMACOTERAPÉUTICO. ¿CONVIENE CONTINUAR LA MEDICACIÓN DURANTE EL PERIOPERATORIO?

Diuréticos

Hipolipemiantes
NO. Se recomienda suspenderlos 24 horas antes o la mañana de la cirugía, para prevenir la hipocalemia (diuréticos tiazídicos o del asa), la hipercalemia (diuréticos ahorradores de potasio) e hipotensión que puede ser de gran magnitud en combinación con los efectos vasodilatadores de los anestésicos $(14,28)$.

NO. Los fibratos y las estatinas pueden producir rabdiomiolisis y miopatía, especialmente en combinación, por ello es aconsejable su discontinuación el día antes de la cirugía. Las resinas de intercambio iónico (colestiramina, colestipol) tampoco se administran el día antes de la cirugía, ya que pueden unirse a algunos medicamentos, disminuyendo su biodisponibilidad (14).

\section{Antitrombóticos}

Antiagregantes plaquetarios (aspirina, ticlopidina, clopidogrel)

Anticoagulantes orales (warfarina, acenocumarol)
NO. Se suspenden 7-9 días antes de la cirugía para evitar complicaciones hemorrágicas. En pacientes con angina inestable o pacientes sometidos a cirugía cardíaca puede resultar beneficioso continuar el tratamiento $(13-15,38,40)$.

Se recomienda ajustar el grado de coagulación, es decir el ratio internacional normalizado (INR) en función de la cirugia. En general, los anticoagulantes orales se suspenden 3-4 días antes de la cirugia programada sustituyéndolos por heparina no fraccionada por vía endovenosa o heparinas de bajo peso molecular por vía subcutánea, en función del riesgo trombótico. Cuando el paciente tolera la ingesta oral se reinicia la anticoagulación oral y cuando se ha estabilizado el grado de coagulación en función de la patología se suspende la heparina $(13,15,38,40,57,58,59)$.

\section{Terapia respiratoria}

\section{Teofilina}

Agonistas $\beta$ adrenérgicos, bromuro de ipratropio y corticosteroides inhalados
Sí. Se deben monitorizar los niveles (14).

Sí. Permiten asegurar la estabilidad respiratoria en pacientes con patologías pulmonares crónicas (14).
Prevención y tratamiento de la osteoporosis

Reemplazamiento hormonal postmenopáusico y Moduladores selectivos de los receptores estrogénicos (tamoxifeno, raloxifeno)

\section{Calcitonina}

Bifosfonatos (alendronato)
NO. Se recomienda discontinuar el tratamiento de 4 a 6 semanas antes de la cirugía para reducir el riesgo de tromboembolismo venoso. En pacientes con cáncer de mama en tratamiento con tamoxifeno, el oncólogo debe valorar el ratio benefico-riesgo $(14,15)$.

Sí. No se han descrito interacciones medicamentosas específicas o contraindicaciones para su uso perioperatorio (15).

NO. La administración de bifosfonatos requiere mantener al paciente erguido 30 minutos tras su administración para evitar la esofagitis por reflujo. La dificultad de cumplir esta premisa en el postoperatorio inmediato hace recomendable su supresión (15). 
ANEXO I (CONT.)

PAUTA A SEGUIR DURANTE EL PERIOPERATORIO CON ALGUNAS DE LAS MEDICACIONES CRÓNICAS MÁS HABITUALES CLASIFICADA POR GRUPO FARMACOTERAPÉUTICO. ¿CONVIENE CONTINUAR LA MEDICACIÓN DURANTE EL PERIOPERATORIO?

Terapia tiroidea

Levotiroxina y antitiroideos (propiltiouracilo, metimazol)

SÍ. En pacientes con hipo o hipertiroidismo el control de la glándula tiroides es fundamental para una cirugía segura. $(14,15,44)$.

Terapia antidiabética

Antidiabéticos orales

Sí, excepto la mañana de la cirugía, para prevenir la aparición de episodios hipoglucémicos. Algunos autores recomiendan suspender la metformina 48-72 horas antes de la cirugía para prevenir el desarrollo de acidosis láctica debida a la acumulación del mismo. Otros autores recomiendan sustituir las sulfonilureas de larga duración por las de corta duración entre 48 y 72 horas antes de la cirugía. Mientras el paciente no tolera la ingesta oral se puede utilizar temporalmente insulina para controlar la glucemia. El tratamiento habitual se reinstaura cuando el paciente reinicia la ingesta oral, o bien cuando la función renal se ha normalizado en los pacientes tratados con metformina, ya que este fármaco está contraindicado en pacientes con I.R. (49,60-65).

Insulina

Sí. La vía de administración depende del tipo de cirugía. En cirugía menor se puede administrar la mitad o dos tercios de la dosis usual de insulina subcutánea (SC). En cirugía mayor se utiliza una infusión de insulina intravenosa (IV) ajustada al estrés y duración de la cirugía y a las necesidades del paciente. La infusión de insulina debe iniciarse a 1 $\mathrm{UI} /$ hora y puede ir ajustándose con incrementos de $0.5 \mathrm{UI} /$ hora en función de la glucemia. La ventaja de la infusión IV respecto a la administración SC es que esta última es más errática y variable. Para prevenir la hipoglucemia, cetosis y acidosis se debe administrar de forma simultánea suero glucosado suplementado con potasio para facilitar la entrada de glucosa en las células. En pacientes con hipercalemia o fallo renal crónico no se deben aportar suplementos de potasio $(14,15,44,48)$.

Fármacos con acción sobre el sistema nervioso

Antiepilépticos y anticonvulsivos

(fenitoina, carbamacepina, ácido valproico)
Sí. Se deben asegurar concentraciones terapéuticas de antiepilépticos y anticonvulsivos para evitar la reaparición de las convulsiones excepto en neurocirugía cuando se quiere extirpar el foco epiléptico. Sus efectos depresores del sistema nervioso central pueden disminuir los requerimientos de anestésicos $(13,14,26,66)$

SÍ, excepto bromocriptina y pergolida que pueden generar excesiva vasodilatación y exacerbar la hipotensión producida por los anestésicos inhalados por lo que no deberían administrarse el día de la cirugía y sé reintroducen cuando el paciente reinicia la ingesta oral $(14,67)$. Se han descrito multiples interacciones entre los antiepilépticos y los fármacos utilizados en la inducción y mantenimiento de la anestesia pero, en general, el beneficio de continuar el tratamiento supera el riesgo de suspenderlo ya que reduce las complicaciones postoperatorias como son la rigidez, acinesia, complicaciones gastrointestinales y respiratorias (67). El factor limitante es la tolerancia oral del paciente. Los únicos fármacos antiparkinsonianos disponibles por vía endovenosa son los anticolinérgicos, que en caso necesario deberán administrarse a la mínima dosis (15). También puede utilizarse apomorfina subcutánea combinada con domperidona rectal (68). A continuación se detallan algunos ejemplos de interacciones clínicamente importantes como la interacción entre levodopa-carbidopa y algunos fármacos usados en anestesia, que puede originar arritmias (13-15,26). Otro ejemplo es la interacción entre selegilina y meperidina que puede ocasionar rigidez, alucinaciones, fiebre, confusión, coma y muerte; así, debería evitarse su uso concomitantemente y monitorizar el uso de otros narcóticos (15). 


\section{ANEXO I (CONT.)}

PAUTA A SEGUIR DURANTE EL PERIOPERATORIO CON ALGUNAS DE LAS MEDICACIONES CRÓNICAS MÁS HABITUALES CLASIFICADA POR GRUPO FARMACOTERAPÉUTICO. ¿CONVIENE CONTINUAR LA MEDICACIÓN DURANTE EL PERIOPERATORIO?

Antidepresivos tricíclicos

Inhibidores selectivos de la recaptación de serotonina (ISRS)

Antidepresivos inhibidores de la monoaminooxidasa (IMAO)

Antipsicóticos

Ansiolíticos (benzodiazepinas)

Analgésicos opiodies

Fármacos contra la "Miastenia Gravis" (piridostigmina, neostigmina)
SÍ. Raramente se han descrito problemas graves relacionados con estos fármacos, aunque la mayoría de autores recomiendan una cautelosa continuación de los mismos durante la cirugía. Debido a sus efectos anticolinérgicos se debe tener especial cuidado cuando se administran con otros medicamentos con dicho efecto $(13-15,40)$.

Sí con precaución. No se han descrito interacciones específicas con los anestésicos, pero se ha publicado algún caso de síndrome serotoninérgico relacionado con la administración concomitante de un ISRS y meperidina y tramadol $(14,15,32)$. También pueden disminuir la agregación plaquetar $(13,40)$.

NO. Los irreversibles (fenelzina, tranilcipromina, isocarboxacida) se deben suspender como mínimo 2 semanas antes de la cirugía para recuperar la actividad de la monoaminooxidasa (MAO) y evitar las multiples interacciones que presenta con otros fármacos (ej: crisis hipertensivas en combinación con simpaticomiméticos, síndrome similar al neuroléptico maligno en combinación con meperidina). Si se requiere continuar el tratamiento, se pueden sustituir por IMAO reversibles de corta duración como meclobemina, que se puede administrar hasta la noche antes de la intervención quirúrgica (13-15,40).

Sí. Las fenotiazinas y otros neurolépticos son relativamente seguros a las dosis habituales. La retirada abrupta de los antipsicóticos puede dar lugar a disquinesia y/o agitación de rebote. Pueden potenciar la depresión del SNC causada por narcóticos y barbitúricos. Raramente causan el síndrome neuroléptico maligno. En pacientes en tratamiento con litio se recomienda continuar y monitorizar los niveles para evitar el rango tóxico $(14,15)$.

Sí. La supresión brusca puede producir el síndrome de retirada especialmente cuando se usan a dosis altas $(14,15)$.

SI. Previenen la debilidad muscular que pudiera alterar la recuperación postquirúrgica (14).

\section{Terapia con corticoides}

Sí. Para evitar los efectos adversos derivados de la supresión del eje hipotalámico-hipofisario-adrenal (EHHA). Los pacientes que se hallan en tratamiento con una dosis igual o inferior a $5 \mathrm{mg}$ / día de prednisona* deben continuar unicamente con la dosis habitual (16) y los que toman una dosis superior a $5 \mathrm{mg} /$ día de prednisona * deben recibir además dosis suplementarias variables según el tipo de cirugía. En cirugía menor se recomienda administrar $25 \mathrm{mg}$ de hidrocortisona* por vía intravenosa (IV) unicamente el día de la cirugía, en cirugía moderada 50-75 mg de hidrocortisona por vía IV el día de la cirugía, disminuyendo hasta la dosis habitual en 1-2 días y en cirugía mayor 100-150 mg de hidrocortisona * por vía IV el día de la cirugía reduciendo hasta la dosis habitual en 1-2 días $(13,40,42,44,45)$.

${ }^{*}$ u otro corticoide a dosis equivalente

Inmunosupresores

Sí. Para minimizar los problemas de rechazo. Se deben monitorizar los niveles. Generalmente no se administran dosis de estrés de corticoides para disminuir la incidencia de sepsis y los problemas de cicatrización de las heridas. En pacientes con sepsis se recomienda disminuir el régimen immunosupresor. La disponibilidad de formulaciones por vía parenteral facilita la continuación, aunque cabe destacar que las formulaciones de tacrolimus y ciclosporina en forma de microemulsión oral tienen una excelente absorción incluso en presencia de íleo postoperatorio $(69,70,71)$.

\section{Antirretrovirales VIH}

Sí. Para minimizar la aparición de resistencias.Se reinstaura de nuevo el tratamiento cuando el paciente tolera la ingesta oral $(14,15)$.

\section{Fitoterapia}

NO. La creencia popular de la inocuidad de las hierbas y otros productos de la denominada medicina alternativa contrasta con la creciente comunicación de interacciones que regularmente van apareciendo con estos productos. Así por ejemplo, la equinácea reduce la efectividad de los inmunosupresores y puede potenciar la hepatotoxicidad de otros fármacos y la efedra puede generar hipertensión y arritmias en combinación con halotano. La presencia de interacciones y efectos desconocidos de estos productos hace recomendable discontinuarlos 1-2 semanas antes de la cirugía (14,15,19). 


\section{Bibliografía}

1. Kluger MT, Gale S, Plummer JL, Owen H. Peri-operative drug prescribing pattern and manufacturer's guidelines. An audit. Anaesthesia 1991; 46 (6): 456-9.

2. Kennedy JM, Van Rij AM, Spears GF, Pettigrew RA, Tucker IG. Polipharmacy in a general surgical unit and consequences of drug withdrawal. Br J Pharmacol 2000; 49: 353-62.

3. Editorial. Risks of interrupting drug treatment before surgery. BMJ 2000; 321: 719-20.

4. Michota FA, Frost SD. Perioperative management of the hospitalized patient. Med Clin N Am 2002; 86:731-48.

5. Roizwn MF. Preoperative evaluation of patiens: a review. Ann Acad Med Singapore 1994; 23 (6): 49-55.

6. Naguib M, Magboul MM, Jaroudi R. Clinically significant drug interactions with general anesthetics--incidence, mechanisms and management. Middle East J Anesthesiol 1997; 14: 127-83.

7. Jin F, Chung F. Minimizing perioperative adverse events in the elderly. Br J Anaesth 2001; 27: 608-24.

8. Selzman CH, Miller SA, Zimmerman MA, Harken AH. The case for beta-adrenergic blockade as prophylaxis against perioperative cardiovascular morbidity and mortality. Arch Sur 2001; 136 (3): 286-90.

9. Goldman L. Evidence-based perioperative risk reduction. Am J Med 2003; 114: 763-4.

10. Armanious S, Wong DT, Etchells E, Higgins P, Chung F. Successful implementation of perioperative beta-blockade utilizing a multidisciplinary approach. Can J Anaesth 2003; 50(2):131-6.

11. Auerbach AD. Goldman MD. b-blockers and reduction of cardiac events in noncardiac surgery. Clinical applications. JAMA 2002; 287 (11): 1445-7.

12. ten Broecke PW, De Hert SG, Mertens E, Adriaensen HF. Effect of preoperative b-blockade on perioperative mortality in coronary surgery. $\mathrm{Br}$ J Anaesth 2003; 90: 27-31.

13. Smith MS, Muir H et Hall R. Perioperative management of drug therapy. Drugs 1996; 51: 238-59.

14. Spell III NO. Stopping and restarting medications in the perioperative period. Med Clin N Am 2001; 85 (5): 1117-28.

15. Mercado DL, Petty BG. Perioperative medication management. Med Clin N Am 2003; 87: 41-57.

16. Ardern DW, Atkinson DR, Fenton AJ. Peri-operative use of oestrogen containing medications and deep vein thrombosis--a national survey. $\mathrm{N}$ Z Med J 2002; 115 (1157): U26.

17. Anómimo. Drug therapy can predict complications in surgical patiens, study suggests. Am J Health-Syst Pharm 2000; 57 (1): 1944.

18. Ang-Lee MK, et al. Herbal medicines and perioperative care. JAMA 2001; 286: 208-16.

19. Skinner CM, Rangasami J. Preoperative use of herbal medicines: a patient survey. Br J Anaesth 2002; 89: 792-5.

20. Crowe S, McKeating K. Delayed emergence and St. John's Wort. Anesthesiology 2002; 96: 1025-7.

21. Vats Da, Noldge-Dchomburg GF. Managing anesthesia in the alcoholic patient. Anaesthesiol Reanim 2002; 27 (6): 160-7.

22. Vorrakitpokatorn P, Limsakul A. Drug-induced hypertermia and rhabdomyolysis during the perioperative period: report of three patients. $\mathrm{J}$ Med Assoc Thai 2002; 85: S884-92.

23. Roure C. Interrupción perioperatoria de la medicación crónica: un riesgo innecesariamente prolongado y probablemente infravalorado. Noticias farmacoterapéuticas 2003; 35: 1-5.

24. Noble DW, Webster J. Interrupting drug therapy in the perioperative period. Drug Saf 2002; 25 (7): 489-95.

25. Goldstein S, Amar D. Pharmacotherapeutic considerations in anesthesia. Heart Dis 2003; 5: 34-48.

26. Anónimo. Drugs in the peri-operative period: stopping or continuing drugs around surgery. DTB 1999; 37: 62-4.

27. Cheng HQ. Contorversies in perioperative medicine. West J Med 1998; 169 (6): 351-5.

28. Sear JW, Higham H. Issues in the perioperative management of the elderly patient with cardiovascular disease. Drus Aging 2002; 19 (6): 429-51.

29. Kozek-Langenercker SA. The effects of drugs used in anaesthesia on platelet membrane receptors and on platelet function. Curr Drug Targets 2002; 3: 247-58
30. Steuer A, Keat AC. Perioperative use of methotrexate - a survey of clinical practice in the UK. Br J Rheumatol 1997; 36: 1009-11.

31. Glass Ps, Howell S, Gan TJ, Ginsberg B. How to manage drug interactions. Eur J Anaesthesiol 1997; 15: 33-9.

32. Tissot TA. Probable meperidine-induced serotonine syndrome in a patient with history of fluoxetine use. Anesthesiology 2003; 98 (6): 1511-2.

33. Crowe S, Collins L. Suxamethonium and donepezil: a cause of prolonged paralysis. Anesthesiology 2003; 98 (2): 574-5.

34. Sanchez J, Demartini A, Roca A. Interaction of donepezil and muscular blockers in Alzheimer's disease. Rev Esp Anestesiol Reanim 2003; 50 (2): 97-100.

35. Dawson J, Karalliedde L. Drug interactions and the clinical anaesthetist. Eur J Anaesthesiol 1998; 15: 172-89.

36. Cammu G. Interactions of neuromuscular blocking drugs. Acta Anaesth Belg 2001; 52: 357-63.

37. Narendran N, Williamson TH. The effects of aspirin and warfarin therapy on haemorrhage in vitreoetinal surgery. Acta Ophthalmol Scand 2003; 81: 38-40.

38. Anónimo. Drugs in the peri-operative period: cardiovascular drugs. DTB 1999; 37: 89-92.

39. Anónimo. Drugs in the peri-operative period: hormonal contraceptives and hormone replacement therapy. DTB 1999; 37: 78-80.

40. Kroenke K, Gooby-Toedt D et Jackson J. Chronic medications in the perioperative period. South Med J; 1998; 91: 358-64.

41. Samama CM, Bastien O, Forestier F, Denninger MH, Isetta C, Juliard $\mathrm{JM}$ et al. Antiplatelet agents in the perioperayive period: expert recommendations of the French Society of Anesthesiology and Intensive Care (SFAR) 2001 -- summary atetement. Can J Anaesth 2002; 49: S26-35.

42. Anónimo. Drugs in the peri-operative period: corticosteroids and therapy for diabetes mellitus. DTB 1999; 37: 68-70.

43. Nicholson G, Burrin JM, Hall GM. Peri-operative steroid supplementation. Anaesthesia 1998; 53: 1091-104.

44. Schiff RL, Welsh GA. Perioperative evaluation and management of the patient with endocrine dysfunction. Med Clin N Am 2003; 87: 175-92.

45. Coursin DB, Wood KE. Corticosteroid supplementation for adrenal insufficiency. JAMA 2002; 287: 236-40.

46. Desborough JP. The stress response to traume and surgery. Br J Anaesth 2000; 85: 109-17.

47. Salem M, Tainsh RE Jr, Bromberg J, Loriaux DL, Chernow B. Perioperative glucocorticoid coverage. A reassessment 42 years after emergence of a problem. Ann Surg 1994; 219 (4): 416-25.

48. Hirsch IB, Paauw DS. Diabetes management in special situations. Endocrinol Metab Clin North Am 1997; 26 (3): 631-45.

49. Scherpereel PA, Tavernier B. Perioperative care of diabetic patients Aur J Anaesthesiol 2001; 18:277-94.

50. Anónimo. Elderly outpatients can discontinue most medications and have no adverse events, study shows, but caution is needed. Am J Health-Syst Pharm 1997; 54: 2787.

51. Ruoff G, Lema M. Strategies in pain management : new and potential indications for COX-2 specific inhibitors. J Pain Symptom Manage 2003; 25 (Supl. 2): S21-31.

52. Shaw M, Mandell BF. Perioperative management of selected problems in patients with rheumatic diseases. Rheum Dis Clin North Am. 1999; 25 (3): 623-38.

53. Grennan DM, Gray J, Loudon J, Fear S. Methotrexate and aerly postoperative complications in patients with rheumatoid arthritis undergoing elective orthopaedic surgery. Ann Rheum Dis 2001; 60: 214-17.

54. Wluka A, Buchbinder R, Hall S et al. Methotrexate and postoperative complications. Ann Rheum Dis 2002; 61:86-7.

55. Jain A, Witbreuk M, Ball C, Nanchahal J. Influence of steroids and methotrexate on wound complications after elective rheumatoid hand and wrist surgery. J Hand Surg 2002; 27: 449-55.

56. Barber L, Barrio J, de Rojas MD, Ibañez F, Ano C, Alepuz R, Montero R. Hipotensión refractaria y sostenida durante una anestesia general asociada al tratamiento crónico con inhibidores de la enzima conversiva de la angiotensina. Rev Esp Anestesiol Reanim 2001; 48 (1): 34-7.

57. Dunn AS, Turpie AG. Perioperative management of patients receiving oral anticoagulants: a systematic review. Arch Intern Med 2003; 163 (8): 901-8. 
58. Kovich O, Otley CC. Perioperative management of anticoagulants and platelet inhibitors for cutaneous surgery: a survey of current practice. Dermatol Sur 2002; 28: 513-7.

59. Protocol "Diagnosi, profilaxi i tractament del tromboembolisme"del Hospital Universitari de Bellvitge.Coordinador:Dr.F Martinez Brotons.4 $4^{\mathrm{a}}$ edició:juny 2001

60. Peters A, Kerner W. Perioperative management of the diabetic patient. Exp Clin Endocrino Diabetes 1995; 103 (4): 213-8.

61. Mercker SK, Maier C, Neuman G, Wulf H. Lactic acidosis as a serious perioperative complication of antidiabetic biguanide medication with metformin. Anesthesiology 1997; 87: 1003-5.

62. Marks JB. Perioperative management of diabetes. Am Fam Physician 2003; 67:93-100.

63. Bailey CJ, Turner R. Metformin. N Engl J Med 1996, 334: 574-9.

64. Lustik SJ, Vogt A, Chhibber AK. Postoperative lactic acidosis in patients receiving metformin. Anesthesiology 1998; 89: 266-7.

65. Jacober SJ, Sowers JR. An update on perioperative management of dia- betes. Arch Inter Med. 1999 Nov 8; 159 (20): 2405-11. Review.

66. Anónimo. When and how to stop antiepileptic drugs in adults. DTB 2003; 41: 41-3.

67. Nicholson G, Pereira AC, Hall GM. Parkinson's disease and anaesthesia. Br J Anaesth 2002; 89: 904-16.

68. Gálvez-Jimenez N, Lang AE. Perioperative problems in parkinson's disease and their management :apomorphine with rectal domperidone. Can J Neurol Sci 1996; 23: 198-203.

69. Testa G, Goldstein R, Toughanipour A, Abbasoglu O, Jeyarajah R, Levy MF, Husberg BS, Gonwa TA, Klintmalm GB. Guidelines for surgical procedures after liver transplantation. Ann Surg 1998; 227: 590-99.

70. Prabhakar G, Testa G, Abbasoglu O, Jeyarajah DR, Goldstein RM, Levey MF, Husberg BS, Gonwa TA, Klintmalm GB. The safety of cardiac operations in the liver transplant recipient. Ann Thorac Surg 1998; 65: 1060-4.

71. Noda H, Fujimura Y, Gohra H, Hamano K, Katoh T, Esato K. Coronary bypass surgery after renal transplantation. Jpn J Thorac Cardiovascular Surg 1999; 47:135-40. 